\title{
$\Theta$ Criterios
}

\section{Los revestimientos externos de la fábrica de la Catedral de Málaga}

Jesús Espinosa Gaitán

Centro de Intervención del IAPH

\section{Resumen}

Las fachadas de la Catedral de Málaga se encuentran revestidas en grandes áreas por una sucesión de revestimientos con distintas coloraciones que se han ido aplicando a lo largo de su historia con intención de embellecer su imagen. En muchas zonas, estos revestimientos se encuentran en un avanzado estado de alteración fundamentalmente a causa de la humedad.

El presente estudio se planteó con el objetivo de conocer la composición y estructura de estos revestimientos y capas de color, y por otro lado establecer posibles factores que han incidido en su estado de conservación. Para ello se ha realizado un estudio mineralógico-petrográfico con el propósito de que los resultados sean de utilidad para establecer la metodología más adecuada para su conservación-restauración. En el estudio se ha establecido que la composición de ciertos niveles de los revestimientos ha sido uno de los principales condicionantes en su alteración. 


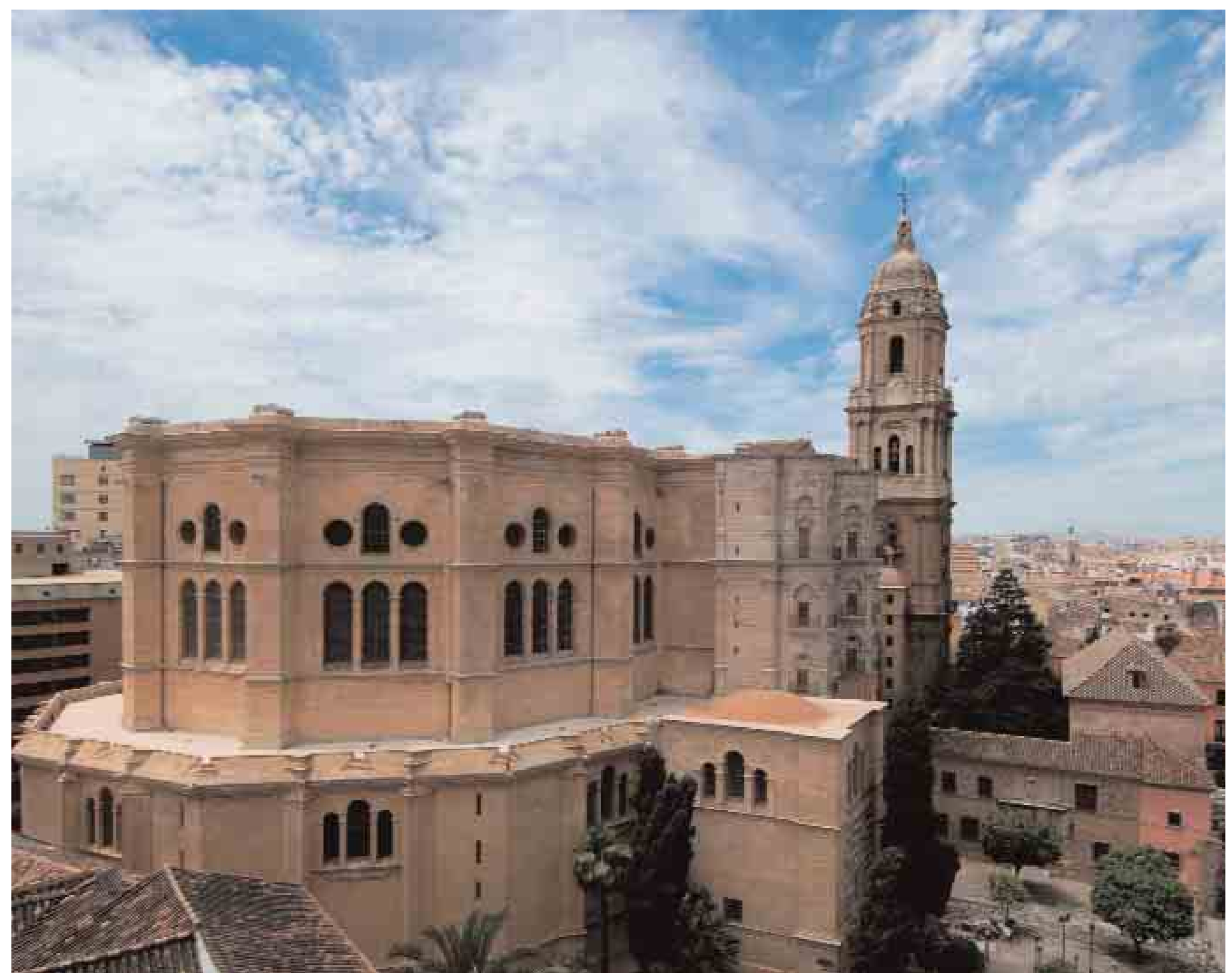

(1) Vista general de la Catedral de Málaga / Eugenıo Fernández, IAPH 


\section{Los revestimientos han ido evolucionando a lo largo de la historia, variando en número, naturaleza y colorido}

\section{INTRODUCCIÓN}

La historia de la construcción de la Catedral de Málaga se prolonga cerca de tres siglos, desde el año 1500 en el que se conciben las primeras ideas de su levantamiento hasta finales del siglo XVIII cuando, agotadas ya todas las posibilidades económicas para terminarla, se suspenden las obras.

En su construcción se emplearon diversos tipos de piedra dependiendo de su función y de la disponibilidad en cada etapa constructiva. Como piedra estructural destacan fundamentalmente dos litotipos: una arenisca de tonos rojos y ocres del Permotrías, que forma parte fundamentalmente de la fachada principal y del primer y segundo cuerpo de las torres, y una roca calcárea bioclástica de tonalidades claras (blancoamarillentas) e importantes variedades en cuanto a su textura y grado de cementación, y que constituye la base de la construcción del tercer y cuarto cuerpo de la Torre Norte, costado Este, y gran parte de los costados Norte y Sur. En menor representación aparece una caliza de tonalidad ligeramente verdosa, y de forma más puntual aparecen otros litotipos como toba calcárea, brecha calcárea, caliza brechoide y mármol blanco y negro (CARRETERO LEÓN, 1993).

La mayoría de estas rocas, especialmente las calizas bioclásticas, se caracterizan por una textura muy heterogénea, especialmente en los bloques de piedra que tienen mayores granulometrías, asociadas por lo general a una menor cementación, presentando un aspecto superficial, en muchos casos, muy grosero, con grandes poros u oquedades. Esto ha propiciado que a lo largo de la historia, y con la intención de embellecer las fachadas de la Catedral, especialmente en las zonas más visibles o donde aparecían los materiales de superficies más irregulares, se aplicaran revestimientos (enfoscados, enlucidos) y capas de color de distinta naturaleza para dar una imagen más homogénea a las superficies externas y acabado superficial de los materiales del monumento.

Estos revestimientos han ido evolucionando a lo largo de la historia del edificio, además de que no son los mismos en todas las zonas, variando en número, naturaleza y coloración ya que fueron fruto de distintas intervenciones, que han propiciado esta distribución.

El paso del tiempo, junto a otros factores de alteración, fundamentalmente la humedad, la acción de partículas contaminantes como el SOz, y la influencia de los aerosoles marinos, entre otros, han hecho que los revestimientos se encuentren en una avanzado de alteración, con notables pérdidas de los mismos, total o parcialmente, además de la formación de costras negras superficiales. Estas pérdidas son mucho más notorias en zonas del edificio que se han visto más afectadas por las humedades, ya sean de origen interno por capilaridad, que afectan básicamente a las zonas bajas de las fachadas, o de origen externo, por efecto de las lluvias o por acumulaciones de humedades en los paramentos por diversos motivos. 


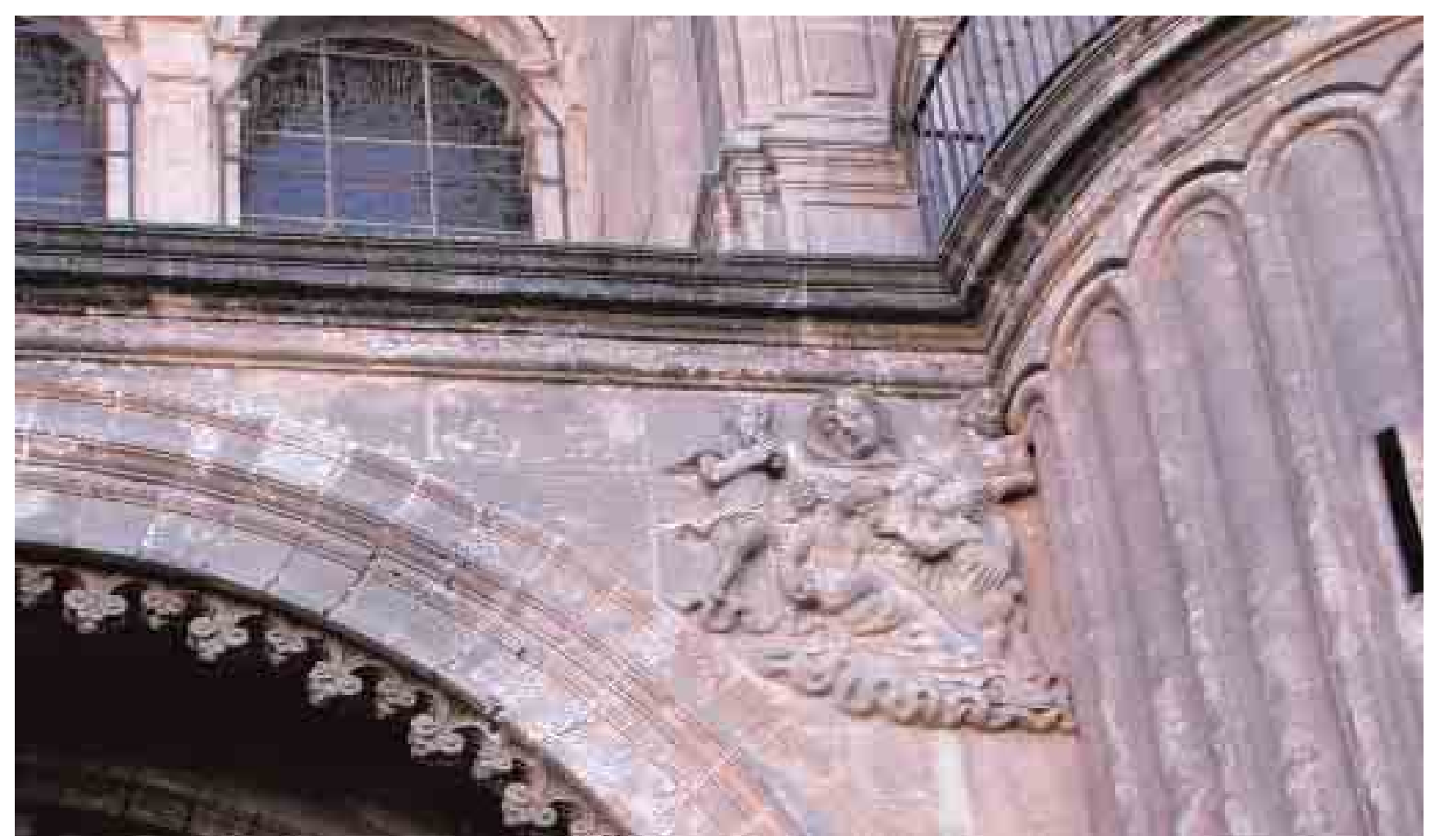

(1) Aspecto de los revestimientos en la zona externa superior de la Portada (Fachada Norte) / MANUEL ALCALDE, IAPH

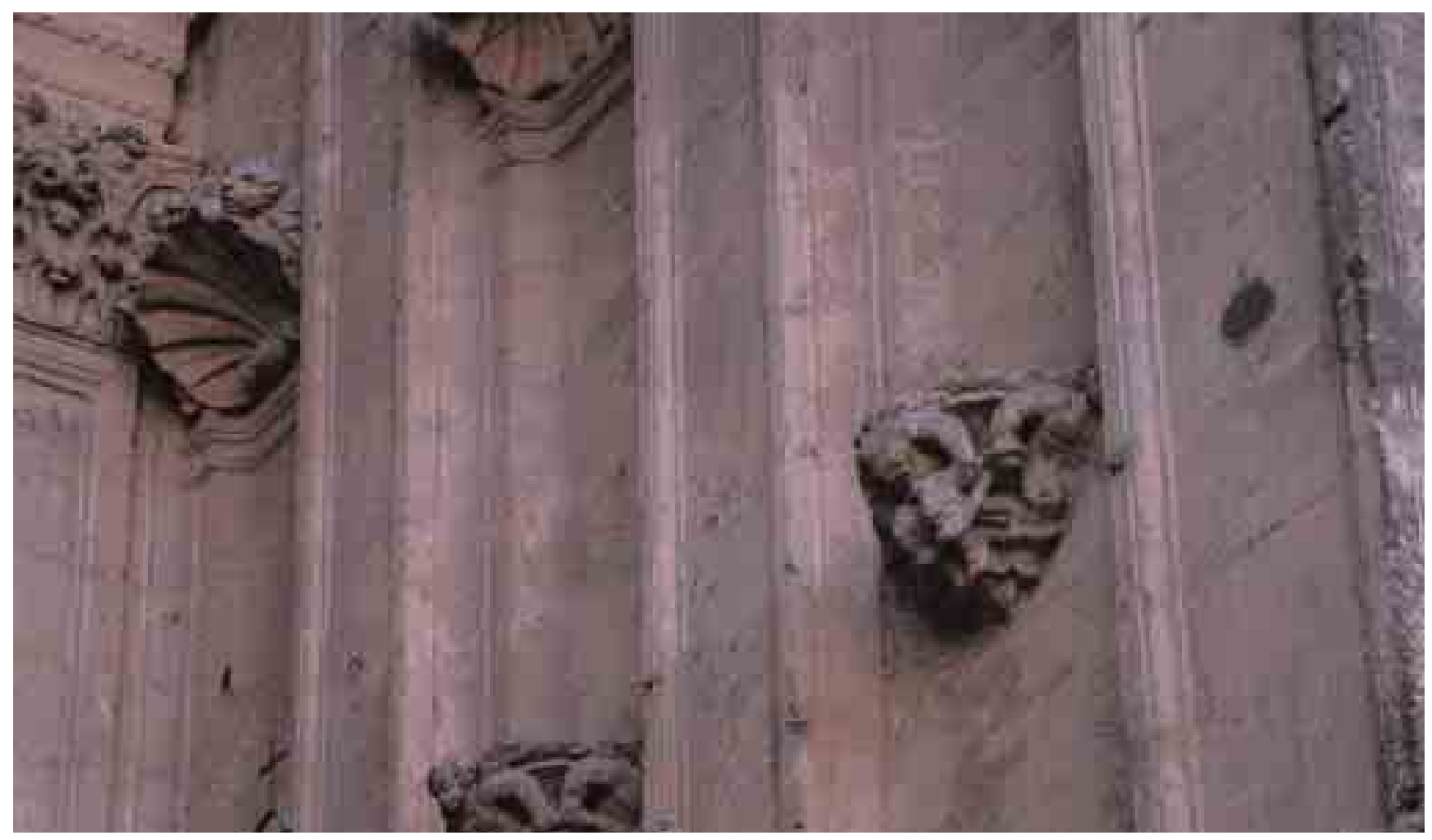

(1) Revestimientos en el interior de la Portada Principal (Fachada Norte) / MANuel ALCALDE, IAPH 


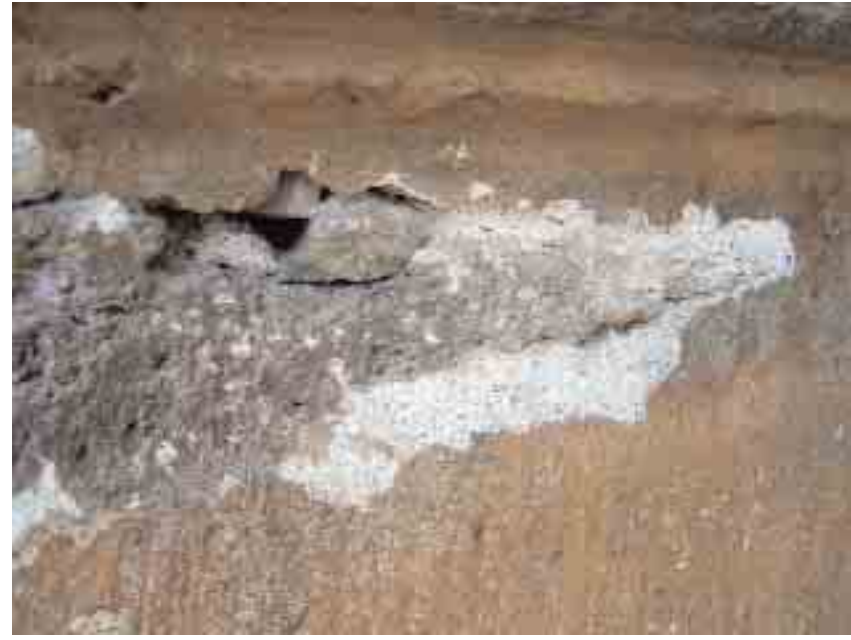

(1) Detalle de la Fachada Norte donde se observa la sucesión de revestimientos / marta García de CASAsola, IAPH

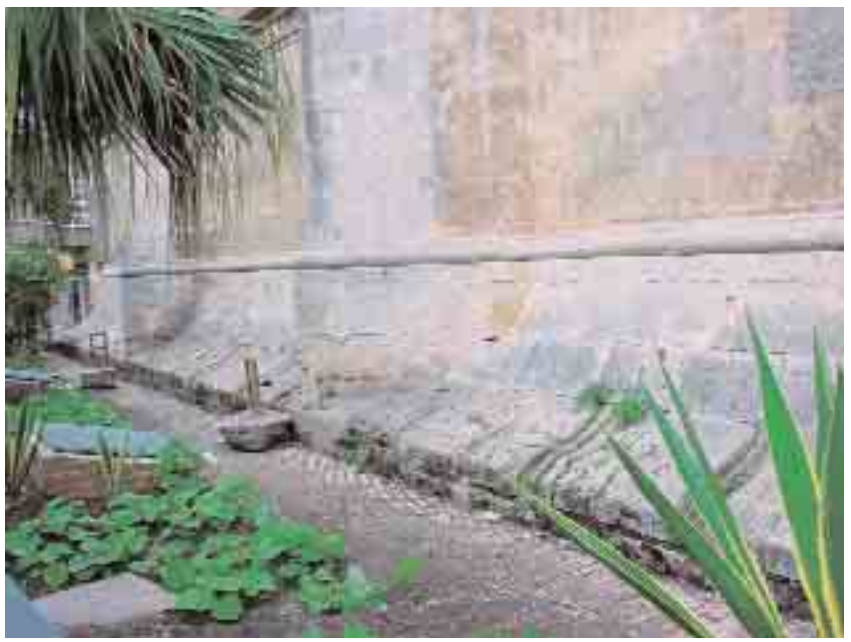

(1) Revestimientos recubriendo las zonas bajas de los muros afectados por la humedad / marta García de Casasola, IAPH

Todo esto ha propiciado la necesidad de realizar un estudio de caracterización de estos revestimientos para establecer su naturaleza y los factores que han podido influenciar en su alteración, con la intención de ayudar a establecer una metodología apropiada en la intervención.

\section{MATERIAL Y MÉTODO}

Se ha estudiado un total de 36 muestras, la mayoría de las cuales pertenece a las portadas principales de la fachadas Norte y Sur de la Catedral. También se tomaron muestras de la zona de la Girola y de revestimientos encontrados en la zona de las terrazas de la fachada Norte en su primer cuerpo.

La mayoría de las muestras incluye toda la sucesión de revestimientos existentes, desde la piedra hasta la capa más superficial, que pueden aparecer en avanzado estado de alteración o en otros casos mejor conservados. También se tomaron muestras de ciertos niveles de forma aislada, tanto de los más internos, que suelen presentar texturas más groseras y espesores mayores (revocos o enlucidos), de niveles intermedios o superiores (coloreados o no), y de las capas superficiales de alteración (incluyendo costras negras) para un estudio más detallado.

Adicionalmente se tomaron muestras de la piedra sobre la que se aplicaron estos revestimientos para estudiar su naturaleza y estado de conservación.

Los métodos de estudio de estas muestras han sido los siguientes:

$\rightarrow$ DRX-Difracción de Rayos X (método de polvo). Con este método se han estudiado muestras obtenidas del raspado superficial de los revestimien- 


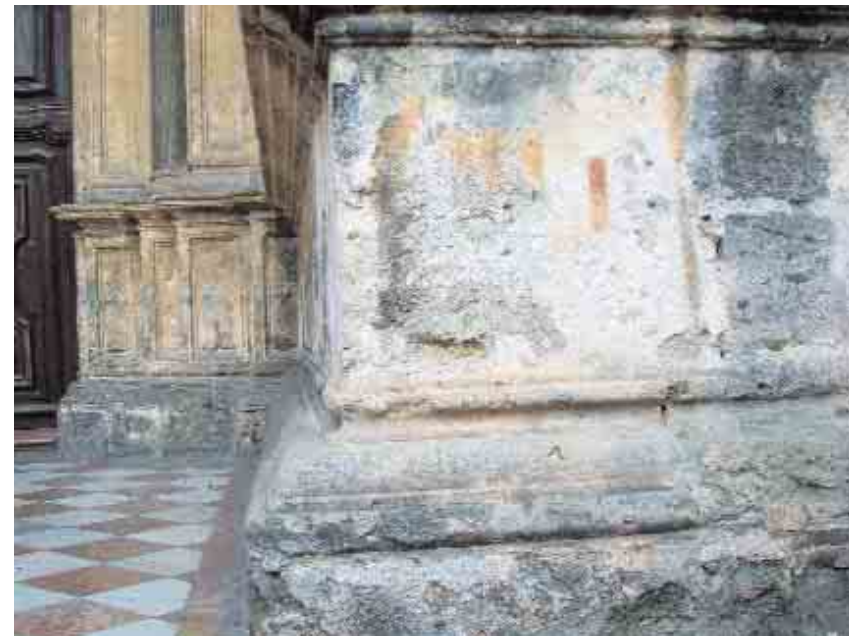

(1) Zona con importantes pérdidas de revestimientos /

MARTA GARCía dE CASASOLA, IAPH

tos coloreados, otras que corresponden a la mezcla de toda la sucesión de revestimientos, dado que por su fino espesor era imposible aislarlos, y otras muestras de revocos y enlucidos en los primeros niveles, cuando era posible su obtención independiente. Esta técnica se emplea para la determinación de la composición mineralógica de cada muestra.

$\rightarrow$ Microscopio petrográfico. Se han estudiado láminas delgadas preparadas en forma de estratigrafía con toda la sucesión de revestimientos y capas de color, previamente embutidas en resina. También se han estudiado láminas delgadas de piedra y de morteros o revocos de mayor espesor. Esta técnica se ha empleado para establecer la estructura y el número de capas en cada una de las muestras, así como la composición mineralógica y textura de cada capa.

$\rightarrow$ Microscopio electrónico de barrido. Se han estudiado las mismas muestras embutidas en resina que se emplearon para la preparación de láminas delgadas. Esta técnica se ha empleado para análisis químicos y observaciones morfológicas de los componentes de los revestimientos.

\section{RESULTADOS}

De los resultados obtenidos con Difracción de Rayos X en muestras de raspado de la capa superficial se obtienen como minerales mayoritarios calcita, cuarzo y yeso. Los contenidos de estos compuestos suelen estar por encima del 15\%, variando sus proporciones entre unas muestras y otras. El algunos casos el compuesto mayoritario es el yeso, pero su procedencia no se puede atribuir únicamente a sulfataciones superficiales por contaminación, tal y como se ha contrastado con las técnicas de microscopía. En cantidades menos importantes aparecen dolomita y filosilicatos. También hay en casi todas las muestras proporcio-

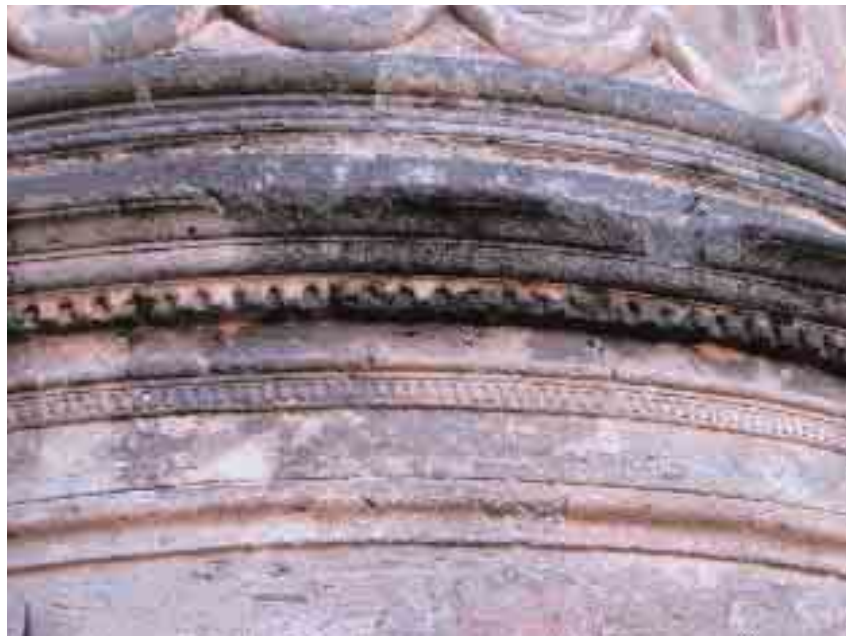

(1) Detalle de los revestimientos /

Marta García de CASASOLA, IAPH

nes pequeñas de óxidos e hidróxidos de Fe, que se atribuyen a los componentes de revestimientos coloreados ocres y rojizos.

La misma composición mineralógica tienen las muestras en las que se han estudiado, mezclados, los revestimientos de fino espesor que presentaba la mayoría de las muestras y que fueron imposibles de aislar. La existencia de yeso en estas muestras (en algunos casos de forma mayoritaria) apunta a la presencia de niveles de yeso o bastardos de yeso y cal en los revestimientos.

Se han detectado en algunas muestras reflexiones de escasa intensidad, que contrastando con las técnicas de microscopía y análisis químicos, se podrían atribuir a melanterita $\left(\mathrm{FeSO}_{4} 7 \mathrm{H}_{2} \mathrm{O}\right)$, que probablemente se ha generado por la alteración y reacción de los compuestos férricos de las capas de color con los sulfatos presentes en los revestimientos.

Las muestras del revoco de mayor grosor, que representa en la mayoría de los casos el primer nivel de la sucesión de los revestimientos, se han estudiado de forma independiente cuando ha sido posible su extracción. Éstos se componen casi en su totalidad de calcita (morteros de cal), junto a cuarzo y filosilicatos, que constituyen el árido. Las muestras de morteros de llagas estudiadas presentan la misma composición.

Las observaciones al Microscopio Óptico han permitido identificar la naturaleza de los niveles de forma individualizada. El estudio de las láminas delgadas de los revestimientos revelan que no hay una misma secuencia en todos los puntos de extracción estudiados. Casi todas las muestras presentan una primera capa correspondiente al revoco blanco de cal. 


\section{Aparecen abundantes niveles con altas proporciones de yeso, además de partículas férricas responsables de las coloraciones ocres/rojizas}

El revoco tiene espesores superiores a 3-4 mm en casi todas las muestras, y se trata de un mortero de cal, de granulometría notoriamente más grosera que los niveles superiores, con tamaño de árido entre 0,5$1 \mathrm{~mm}$, que se constituye de fragmentos de rocas de distinta naturaleza, fundamentalmente rocas de estructura pizarrosa, con elevado contenido en micas negras.

Por encima del revoco, o aplicado directamente sobre la piedra en aquellas zonas donde ésta posee texturas más finas, aparece un enlucido de color amarillento de espesor más fino, entre 1-2 mm, que también es de cal, de menor proporción de árido y menor granulometría.

Sobre estos materiales comienza una sucesión de finas capas de enlucidos o capas de color que pueden ser de diferente naturaleza y disposición en la sucesión.

En las tablas 1, 2 y 3 se recogen de forma resumida los resultados de algunas de las muestras más representativas en cada una de las zonas del edificio estudiadas.

Suele ser común en las capas de fino espesor la presencia de partículas que normalmente dan coloraciones de ocres a rojizas; estas partículas o agregados anaranjados aparecen en diferentes proporciones dependiendo de las capas y de las muestras. En otras muestras se ha podido observar que la coloración es debida a la presencia en la matriz de polvo arcilloso rico en compuestos férricos mezclado con cal o con yeso. En algunas capas no existen partículas colorantes y se componen sólo de yeso y/o de cal.

En casi todas las muestras que contienen niveles de yeso se observan disoluciones y grandes pérdidas de algunos de estos niveles, y cómo esto afecta al conjunto de las capas, a las capas de cal, o incluso a la piedra interior.

Mediante Microscopía Electrónica se han realizado análisis globales de cada una de las capas, para establecer sus composiciones químicas mayoritarias, así como análisis puntuales sobre las partículas colorantes. Los resultados están en concordancia con los obtenidos con las otras técnicas. En el caso del revoco blanco aparecen composiciones atribuibles a cal (Ca) y a los áridos silícicos (Si, $\mathrm{Al}, \mathrm{Mg}, \mathrm{Fe}$ ). El enlucido amarillento presenta composiciones elevadas de calcio e importantes contenidos de hierro atribuibles a los glomérulos anaranjados.

En el resto de los niveles aparecen composiciones a base de azufre y/o calcio, dependiendo de la muestra, y corresponderán a niveles de yeso, cal o bastardos. En algunos casos se observan composiciones de silicio, aluminio, hierro y calcio, indicativo de mezcla de arcillas ferruginosas. 
Tabla 1. Cuadro resumen (microscopía óptica) de algunas muestras de la Portada Principal de la Fachada Norte

\begin{tabular}{|c|c|c|c|c|c|c|c|c|c|c|c|c|c|c|c|c|}
\hline \multirow[b]{2}{*}{ Capa } & \multicolumn{4}{|c|}{ СТМ-3 } & \multicolumn{4}{|c|}{ СтМ-5 } & \multicolumn{4}{|c|}{ СТМ-10 } & \multicolumn{4}{|c|}{ СТМ-14 } \\
\hline & Esp & Mtr & Prt & Estd & Esp & Mtr & Prt & Estd & Esp & Mtr & Prt & Estd & Esp & Mtr & Prt & Estd \\
\hline 1 & $4-5$ & RB & $\mathrm{RB}$ & B & 3 & $\mathrm{RB}$ & $\mathrm{RB}$ & $\mathrm{R}$ & 1.5 & EA & EA & $\mathrm{R}$ & 6 & $\mathrm{RB}$ & $\mathrm{RB}$ & B \\
\hline 2 & 1.5 & $E A$ & EA & B & 1.5 & $E A$ & EA & $\mathrm{R}$ & 0.75 & B & GN & $\mathrm{R}$ & 0.13 & B & GN & B \\
\hline 3 & 0.20 & B & GN & $\mathrm{R}$ & 0.25 & C & - & $\mathrm{R}$ & 0.07 & C & & $\mathrm{R}$ & 0.17 & $\mathrm{Y}-\mathrm{A}$ & GN & B \\
\hline 4 & 0.15 & $Y$ & & $M$ & 0.15 & $Y$ & PF & $M$ & 0.1 & B & GN-PF & $M$ & 0.17 & $\mathrm{Y}$ & $\mathrm{PF}$ & $\mathrm{R}$ \\
\hline 5 & 0.08 & C-A & $\mathrm{PF}$ & $M$ & 0.1 & $\mathrm{Y}$ & GN-PF & $\mathrm{R}$ & 0.05 & $Y$ & PF-ML & $M$ & 0.025 & C & & $M$ \\
\hline 6 & 0.05 & C & PF & $M$ & 0.1 & C & & $\mathrm{R}$ & & & & & & & & \\
\hline 7 & & & & & 0.25 & $Y$ & PF & $\mathrm{R}$ & & & & & & & & \\
\hline 8 & & & & & 0.25 & $Y$ & PF-ML & $M$ & & & & & & & & \\
\hline
\end{tabular}

Tabla 2. Cuadro resumen (microscopía óptica) de algunas muestras de la Portada Principal de la Fachada Sur

\begin{tabular}{|c|c|c|c|c|c|c|c|c|c|c|c|c|c|c|c|c|}
\hline \multirow[b]{2}{*}{ Capa } & \multicolumn{4}{|c|}{ СТM-19 } & \multicolumn{4}{|c|}{ СТМ-21 } & \multicolumn{4}{|c|}{ СТM-22 } & \multicolumn{4}{|c|}{ СTM-36 } \\
\hline & Esp & Mtr & Prt & Estd & Esp & Mtr & Prt & Estd & Esp & Mtr & Prt & Estd & Esp & Mtr & Prt & Estd \\
\hline 1 & $4-5$ & $\mathrm{RB}$ & $\mathrm{RB}$ & B & 1 & $\mathrm{RB}$ & $\mathrm{RB}$ & B & 1.5 & $\mathrm{RB}$ & RB & B & 0.05 & $Y$ & PF & $\mathrm{R}$ \\
\hline 2 & 0.1 & $B$ & $\mathrm{PF}$ & $M$ & 1.5 & $E A$ & EA & $M$ & 0.75 & EA & EA & $\mathrm{R}$ & 2 & $\mathrm{RB}$ & $\mathrm{RB}$ & $\mathrm{R}$ \\
\hline 3 & 0.03 & C & - & $M$ & 0.25 & $\mathrm{Y}$ & $\mathrm{PF}$ & M & 0.07 & $Y$ & GN-PF & $\mathrm{R}$ & 0.05 & $\mathrm{Y}$ & $\mathrm{PF}$ & $\mathrm{R}$ \\
\hline 4 & 0.04 & $Y$ & PF & $M$ & & & & & 0.05 & C & GN & $M$ & 0.03 & C & & $\mathrm{R}$ \\
\hline 5 & & & & & & & & & & & & & 0.08 & $Y$ & GN & $\mathrm{R}$ \\
\hline
\end{tabular}

Tabla 3. Cuadro resumen (microscopía óptica) de algunas muestras de la Zona de la Girola y del Segundo Cuerpo (terrazas)

\begin{tabular}{|c|c|c|c|c|c|c|c|c|c|c|c|c|c|c|c|c|}
\hline \multirow[b]{2}{*}{ Capa } & \multicolumn{4}{|c|}{ CTM-25 (Girola) } & \multicolumn{4}{|c|}{ CTM-33 (Girola) } & \multicolumn{4}{|c|}{ CTM-26 (segundo cuerpo) } & \multicolumn{4}{|c|}{ CTM-24 (Girola) } \\
\hline & Esp & Mtr & Prt & Estd & Esp & Mtr & Prt & Estd & Esp & Mtr & Prt & Estd & Esp & Mtr & Prt & Estd \\
\hline 1 & 2 & $\mathrm{EA}$ & EA & B & 3 & $\mathrm{RB}$ & $\mathrm{RB}$ & B & 0.062 & C & GN & B & 0.75 & B & $\mathrm{PF}$ & B \\
\hline 2 & 0.2 & B & & $\mathrm{R}$ & 1 & EA & EA & $\mathrm{R}$ & 0.3 & c & & B & 0.4 & B & $\mathrm{GN}$ & $\mathrm{R}$ \\
\hline 3 & 0.05 & C-A & PF & $\mathrm{R}$ & 0.12 & Y & & $\mathrm{R}$ & 0.12 & c & & B & 0.15 & Y & $\mathrm{PF}$ & $\mathrm{R}$ \\
\hline 4 & 0.25 & Y & PF & M & 0.025 & c & $\mathrm{PF}$ & M & 0.1 & B & & B & 0.3 & C & & B \\
\hline 5 & 0.03 & C-A & PF & $\mathrm{R}$ & & & & & 0.25 & Y & PF & $\mathrm{R}$ & 0.15 & C & & M \\
\hline 6 & 0.02 & Y & & $M$ & & & & & & a 11 са & de C, & & 0.1 & B & & $M$ \\
\hline
\end{tabular}

Esp: espesor de capa (mm); Mtr: Matriz de la capa; RB: Revoco blanco; EA: Enlucido amarillento; B: bastardo, mezcla cal y yeso; Y: yeso; C: cal; A: arcillas rojizas; Prt: partículas o agregados en las capas; GN: glomérulos naranjas; PF: partículas férricas; ML: melanterita; Estd: estado de conservación de cada capa; B: estado bueno; R: estado regular; M: mal estado

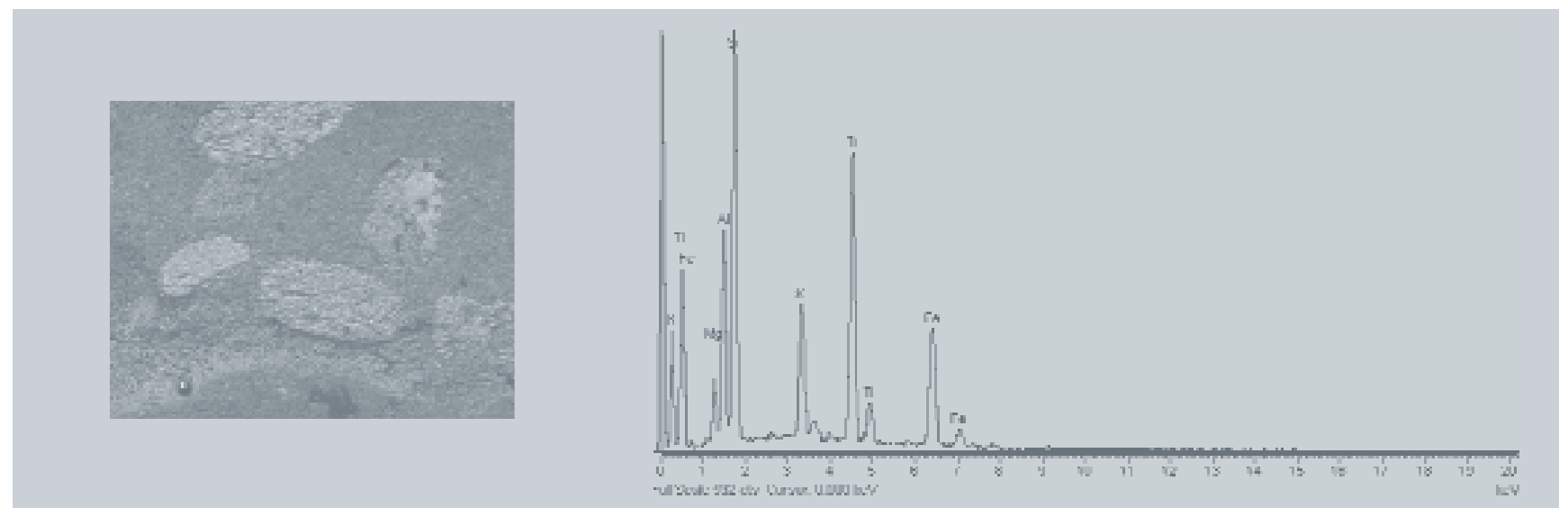

๑ CTM-5. Espectro análisis SEM-EDX composición de grano en capa enlucido (IImenita-Rutilo) 


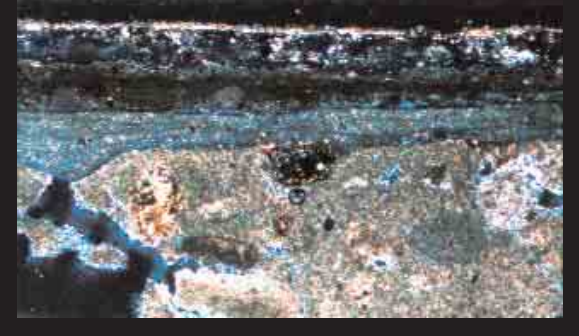

(1) Microscopía óptica. Sucesión de capas muestra CTM-14. Objetivo 5x / Jesús EsPINOSA, IAPH

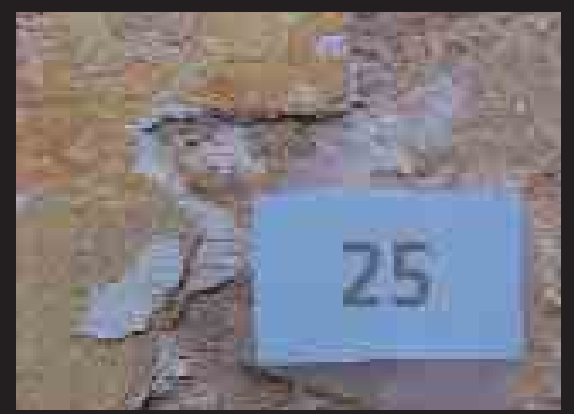

(1) Punto de extracción de la muestra CTM-25 / JESÚs ESPINOSA, IAPH

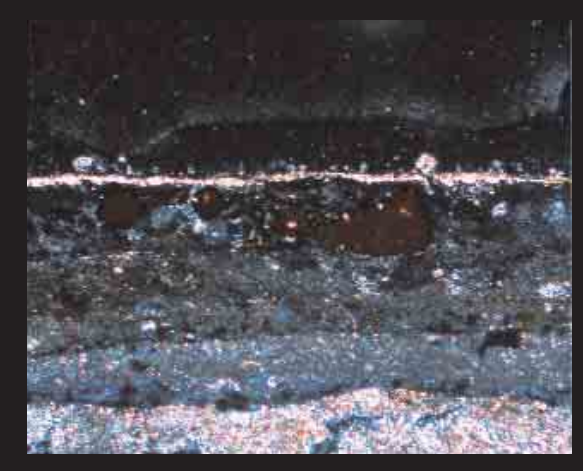

(1) Detalle de las últimas capas con partículas

pigmentantes. Objetivo 10x / JEsús EsPINOSA, IAPH

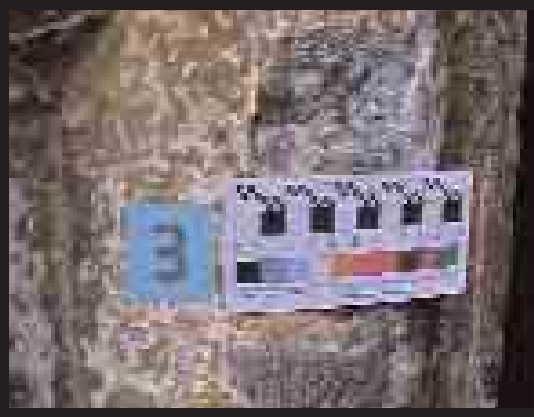

(1) Zona de extracción de la muestra CTM-3 (Portada Norte) JESÚS ESPINOSA, IAPH

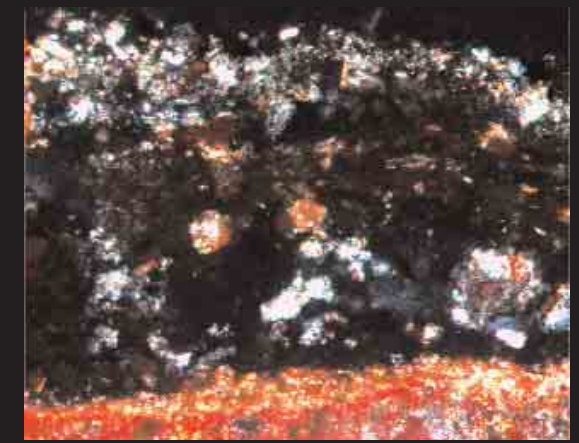

(1) CTM-7. Aspecto de la alteración de las tres últimas capas con partículas de coloración y yeso. Objetivo 20x / JESÚS ESPINOSA, IAPH

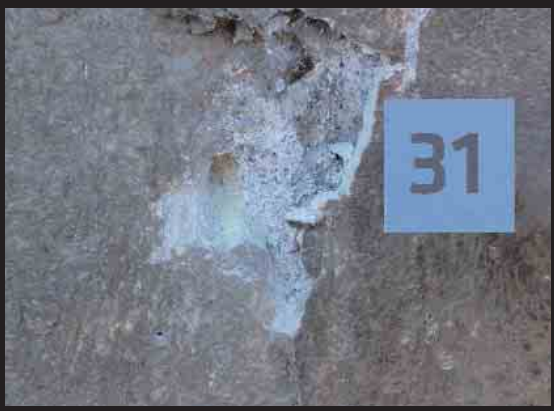

(1) Punto de extracción de la muestra CTM-31 (Girola) / Manuel AlCALDE, IAPH 
En cuanto a las partículas o agregados de estas capas, hay que señalar que la mayoría de ellas corresponde a fases férricas (óxidos o oxihidróxidos de Fe), pero algunas de ellas además de hierro presentan importantes contenidos de titanio.

Se debe mencionar que se han detectado en algunas muestras cantidades importantes de cloro y sodio (halita), efecto de los aerosoles marinos. En algunas muestras se han observado cristales en superficie que se componen básicamente de hierro y azufre (probablemente melanterita).

Las muestras de materiales pétreos estudiadas corresponden todas a calizas bioclásticas (ostrácodos, equinodermos, algas rojas, nummulites, briozoos y serpúlidos), con una textura condicionada, básicamente, por el tamaño de los bioclastos y el grado de cementación sedimentaria que contengan. En algunas de ellas el grado de cementación se ha visto modificado respecto al original por fenómenos de disolución tras su puesta en obra, lo que ha favorecido un incremento en la porosidad. El tamaño habitual de los bioclastos es de tipo arena media-gruesa, entre 1,5-3 mm, aunque existen tamaños inferiores $y$, en algunos casos, de tamaño grava (> $3 \mathrm{~mm}$ ). Dependiendo de la abundancia de unos u otros tamaños de bioclastos en los bancos de sedimentación, la roca poseerá texturas más groseras o finas. Igualmente, dependiendo del grado de cementación, la roca tendrá mayor o menor compactación y porosidad. Se observa a grandes rasgos que, a menor granulometría, mayor grado de cementación en la roca y menor macroporosidad.

En algunas zonas se han observado en los poros depósitos de yeso, que podría ser intrínseco al material pétreo o proceder de los revestimientos más externos que contienen yeso.

\section{CONCLUSIONES}

En casi todas las muestras se ha establecido una sucesión de revestimientos (coloreados o no) variables en naturaleza y número, pero no se puede establecer una serie común para la globalidad de las muestras, aún siquiera para muestras extraídas de una misma zona del edificio.

Este hecho puede estar condicionado por factores como la pérdida por alteración de determinadas capas antiguas antes de la aplicación de otras posteriores, pérdidas más recientes de los últimos niveles, o bien porque las distintas zonas del edificio no hayan sido objeto de las mismas actuaciones a lo largo de su historia.

En cualquier caso y a grandes rasgos, sí parece generalizado que la primera intervención para cubrir la piedra de la fábrica consistió en la aplicación de un revoco blanco de cal, de textura grosera, con áridos de fragmentos de roca de diversa naturaleza (esquistos, pizarras, areniscas). Éste parece haberse aplicado fundamentalmente en las zonas donde la piedra subyacente aparecía con una superficie muy irregular, con grandes oquedades y escasa cementación. Este revoco se usaría con la intención de homogeneizar o enrasar las superficies antes de la aplicación de revestimientos más finos y capas de color.

En gran número de muestras correspondientes a distintas zonas del edificio se ha encontrado antes de las capas de más fino grosor, un enlucido de textura más fina que el revoco blanco, de un grosor que oscila entre 1-2 mm, y de coloración amarillenta. Este enlucido se compone básicamente de cal, con muy escaso árido silíceo, y contiene partículas o pequeños agregados de color anaranjado (que le confieren el color al enlucido), y que tienen hábitos cristalinos similares a los carbonatos, por lo que pudiera tratarse de carbonatos ricos en hierro. Este enlucido puede aparecer sobre el revoco blanco o directamente sobre la piedra, donde ésta no aparece con tantas irregularidades superficiales, y pudo aplicarse como nivel de preparación de las capas coloreadas más finas.

Sin embargo, existen algunas muestras en las que este enlucido no se ha observado y las capas coloreadas aparecen directamente sobre la piedra o directamente sobre el revoco blanco.

A partir de esta base, comienza una sucesión de finos revestimientos, en la mayoría de los casos con partículas pigmentantes, alternados en algunos casos con capas sin coloración. La sucesión de estas capas es muy variable de unas muestras a otras, tanto en número, naturaleza y pigmentación de las mismas, espesor, así como la secuencia de aparición de los distintos niveles. Existen muestras en las que por efectos de la alteración se observan sólo restos mezclados de todas las capas.

Un hecho generalizado en el caso de los revestimientos coloreados es la utilización indistinta de cal, yeso, o mezcla de ambos, como base aglomerante de las partículas pigmentantes férricas, y de forma menos abundante también se utiliza una mezcla de polvo arcilloso rico en compuestos férricos con yeso o cal. En otras muestras aparecen niveles intercalados o de terminación sin pigmentos, compuestos exclusivamente de cal o de yeso.

La naturaleza de las partículas pigmentantes que confiere la gama de colores ocres a rojizos de los revestimientos es fundamentalmente de base de compuestos de hierro, concretamente óxidos e hidróxidos de hierro, representados por fases férricas desde Hematites ( $\mathrm{Fe}_{2} \mathrm{O}_{3}$ ), para las tonalidades más pardorojizas, a Goetita y Lepidocrocita (FeO.OH), con tonalidades más pardas o pardo amarillentas, o la asociación de estos minerales con distintos grados de hidratación (limonita), con distintas coloraciones dentro de la misma gama. Los análisis también señalan la presencia de partículas que contienen titanio que corresponderían a ilmenita y/o rutilo.

Independientemente de la distribución y secuencia en la que aparezcan las capas, en casi todas las muestras se han detectado niveles de yeso 


\section{La elevada proporción de yeso puede considerarse uno de los principales factores que han propiciado el deterioro de los revestimientos}

o mezclas de cal y yeso. Se ha observado, casi de forma generalizada, que las capas que contienen yeso están bastante alteradas, a la vez que favorece y acelera la alteración del resto de revestimientos que integran la sucesión.

Aunque la solubilidad y movilidad del yeso no es tan alta como la de otras sales solubles, el hecho de presentarse en tan elevadas proporciones puede considerarse uno de los principales factores que han propiciado la evolución del deterioro de los revestimientos.

El yeso, en presencia constante de humedad, o en contacto durante largos periodos, tiende a disolverse y movilizarse, fundamentalmente hacia el exterior, pero también puede hacerlo hacia el interior de los materiales pétreos, tal y como se ha visto en algunas muestras estudiadas.

El problema de la existencia de yeso en los revestimientos se hace especialmente grave en las partes bajas del edificio. En estas zonas, la existencia de revestimientos sobre la piedra supone una barrera para la transpiración de las aguas capilares ya que estos materiales son menos porosos, y por tanto, con menor velocidad de transpiración o de secado. Esta situación favorece que la humedad permanezca más tiempo en la interfase piedra-revestimientos, propiciándose los fenómenos de disolución y reprecipitación de sales solubles, tanto procedentes del interior, movilizados desde el suelo y de la propia piedra, o de los revestimientos que se encuentran en contacto con el agua.

El hecho de que el yeso sea un compuesto relativamente soluble incrementa los efectos de estos procesos, provocando la formación de oquedades internas por pérdidas de material, recristalizando especialmente hacia la superficie. Esto ocasiona que en algunos casos los revestimientos aparezcan descohesionados en las capas internas $y$, finalmente, tiendan a desprenderse por desplome.

De esto se deduce que la presencia del yeso como material constituyente en los revestimientos supone un problema al plantearse los métodos de conservación de los mismos, especialmente en las zonas bajas del edificio, en las que es difícil evitar el contacto de estos materiales, por su cara interna, con las aguas capilares procedentes del sustrato.

Si no se evita el contacto continuo o cíclico de la humedad interna con los revestimientos, aunque se consoliden e hidrofugen superficialmente, el problema no se solucionaría, sino que es probable que a corto plazo se pueda agravar ya que la aplicación de dichos productos podría ralentizar aún más la evaporación de las aguas capilares.

En zonas del edificio que no se vean afectadas por la acción de las aguas capilares es recomendable la protección de estos revestimientos para evitar o aminorar la posible entrada de agua desde el exterior. 


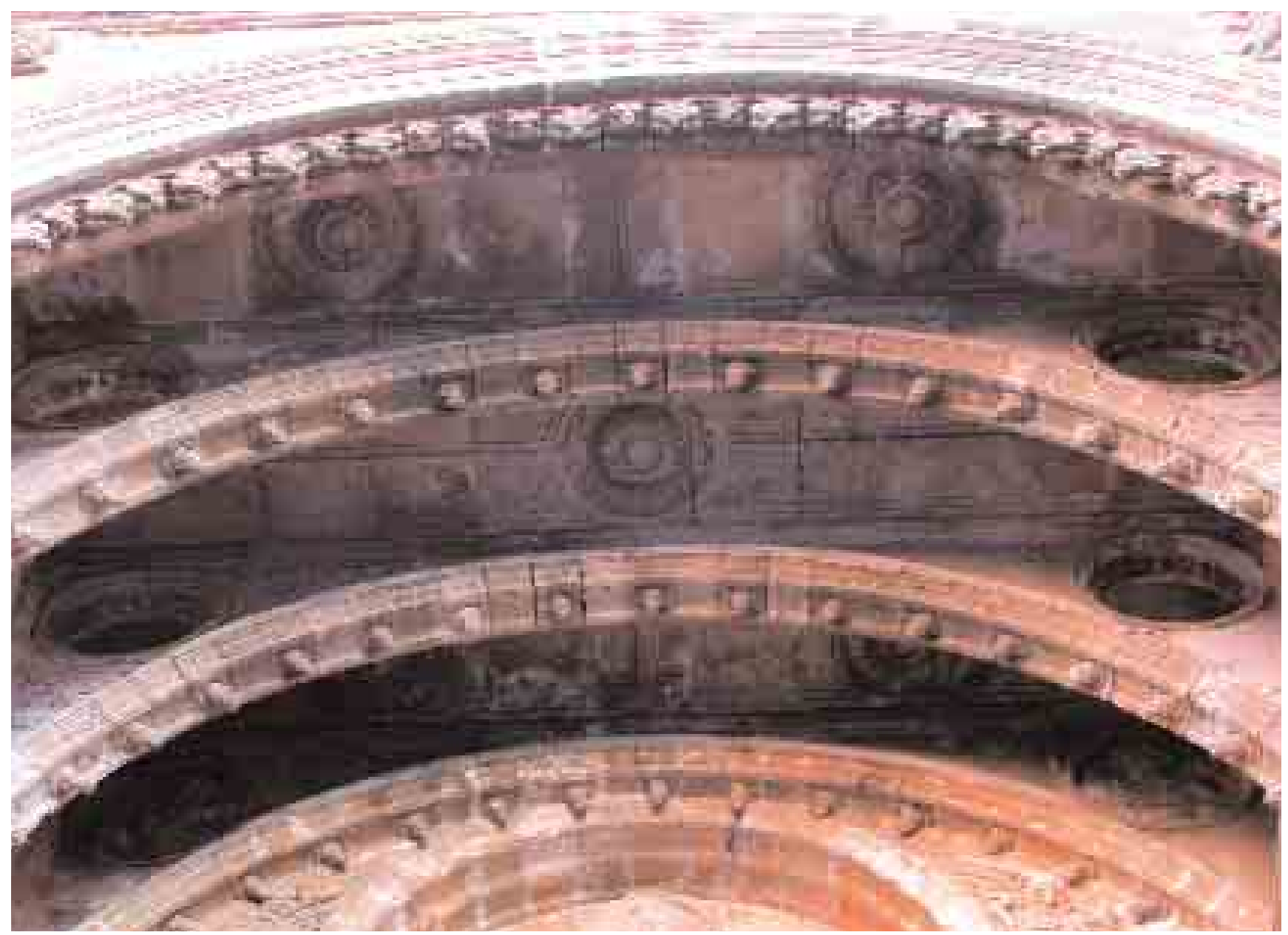

(1) Revestimientos en el interior de la Portada Principal (Fachada Norte) / MANUEL ALCALDE, IAPH

\section{Bibliografía}

ADAMS, A.E.; MACKENZIE, W.S.; GUILFORD, C. (1984) Atlas of Sedimentary Rocks Under the Microscope. Essex: Longman Scientific \& Technical, 1984

ASHURST, J.; ASHURST, N. (1988) Mortars, Plasters and Renders. Practical Building Conservation Series, v. 3. Gower Technical Press Ltd. England, 1988

BERDUCOU, M.C. (coord.) (1990) La conservation en archéologie Paris: Ed. Masson, 1990

CARRETERo LEÓN, M.I. (1993) La piedra de la Catedral de Málaga, estado de alteración y tratamientos de conservación. Tesis doctoral. Universidad de Sevilla (inédita)

FoLK, R.L. (1974) Petrology of Sedimentary Rocks. Austin, Texas: Hemphills, 1974
GONZÁLEZ LIMÓN, T.; ÁLVAREZ DE BUERGO BALLESTER, M. (2002) Los revocos de cal de las fachadas de la Plaza de la Corredera de Córdoba. Materiales de Construcción, v. 52, n² 267, pp. 19-30

GROSSI, C.M.; ESBERT, R.M. (1994) Las sales solubles en el deterioro de rocas monumentales. Materiales de Construcción, v. 44, $n^{\circ} 235$, pp. 15-30

KLEIN, C.; HURLBURT, C.S. (1982) Manual de Mineralogía de Dana. Barcelona: Reverté, S.A., 1982

MACKENZIE W.S.; GUILFORD, C. (1994) Atlas of Rock-forming Minerals in thin Section. Essex: Longman Scientific \& Technical, 1994

MONTAGNA, G. (1993) I pigmenti. Prontuario per l'arte e il restauro. Firenze: Nardini Editore, 1993
PUERTAS, F.; BLANCO VARELA, M.T.; PALOMO, A. (1994) Estucos y hormigones romanos de la ciudad de Baelo Claudla (Cádiz): caracterización y causas de deterioro. Materiales de Construcción, v. 44, $n^{\circ}$ 236, pp. 15-30

SculuY, J.C. (1990) The Fundamentals of corrosion. Oxford: Pergamon Press, 1990

SEBASTIÁN PARDO, E.; RODRÍGUEZ NAVARRO, C. (1996) "Formación de costras negras en materiales pétreos ornamentales". Revista Ajimez, 1, pp. 4-12 